\title{
SELECTION OF PROMISING RAPESEED MUTANTS THROUGH MULTI-LOCATION TRIALS
}

\author{
MA MALEK* ${ }^{1}$, MR ISMAIL ${ }^{1}$, FI Monshi ${ }^{2}$, MMA MONDAL ${ }^{3}$ AND MN Alam \\ Plant Breeding Division, Bangladesh Institute of Nuclear Agriculture, \\ Mymensingh-2202, Bangladesh
}

Key words: Mutant, Rapeseed, Evaluation, Farmers’ field trial

\begin{abstract}
Significant variations were observed for most of the characters among the three promising $\mathrm{M}_{5}$ rapeseed mutants. Mutant RM-025 produced the highest seed yield of $1945 \mathrm{~kg} / \mathrm{ha}$ followed by RM-005 (1892 kg/ha) and had 11.6 and $8.5 \%$ higher seed yield, respectively than the control variety. Under farmers' management, on an average, mutant RM-025 and RM-005 produced 1824 and $1725 \mathrm{~kg} /$ ha with $11.3 \%$ and $5.2 \%$ higher seed yield, respectively than the control variety. These two mutants also had higher number of primary branches and siliquae/plant, and 1000-seed weight and required shorter maturity period than the control variety under both management practices.
\end{abstract}

Oleiferous Brassica (rapeseed and mustard) is an important first ranking oilseed crop in Bangladesh. But its production per unit area is very low i.e., $902 \mathrm{~kg} / \mathrm{ha}$ (BBS 2010). There are many factors responsible for its low yield but the most important one is the non-availability of high yielding varieties. It is therefore, imperative to develop high yielding varieties to meet the local production of edible oil. The present study was undertaken to evaluate the performance of the newly developed promising mutants of Brassica napus L. for their seed yield and yield components at four different agro-ecological zones of Bangladesh under two management practices and finally to select the promising mutant(s) to be registered as variety.

Homogenous seeds of Binasarisha-4 of B. napus were irradiated with 500, 600, 700 and 800 Gy doses of gamma rays using $\mathrm{Co}^{60}$ gamma cell to induce new genetic variability and planted $\mathrm{M}_{1}$ generation at Bangladesh Institute of Nuclear Agriculture (BINA), Mymensingh in 2005 for the selection of improved genotypes in the subsequent generations. Selection for the desired agronomic traits was carried out in $\mathrm{M}_{2}, \mathrm{M}_{3}$ and $\mathrm{M}_{4}$ through trials. From the mutant population, three $\mathrm{M}_{5}$ mutants (RM-005, RM-025 and RM2-16-96) were finally selected on the basis of their promising performance for seed yield and yield contributing characters with shorter maturity period.

Experiments with three $\mathrm{M}_{5}$ mutants of rapeseed was carried out in the experimental farms of BINA and its sub-stations at Magura, Ishurdi and Rangpur. On-station trial in annex farm at Jamalpur using research management while on-farm trial for farmers' management was conducted in the farmers' field at Mymensingh, Rangpur, Magura and Pabna districts during October, 2009 to February, 2010. RCBD with four replications in unit plot size $28.8 \mathrm{~m}^{2}(6.0 \mathrm{~m} \times 4.8 \mathrm{~m})$ having $30 \mathrm{~cm}$ spacing for row to row and $6-8 \mathrm{~cm}$ for plant to plant in a row was followed. Seeds were sown in optimum time between 25 October and 10 November, 2009. Research management comprised of recommended management packages for on-station trial while farmers' practice comprised of recommended doses of fertilizers with poor cultural management done by the

*Author for correspondence: $<$ mamalekbina@yahoo.com>. ${ }^{1}$ Institute of Tropical Agriculture, Universiti Putra Malaysia, 43400 UPM Serdang, Selangor, Malaysia. ${ }^{2}$ Department of Genetics and Plant Breeding, Sylhet Agricultural University, Sylhet-3100, Bangladesh. ${ }^{3}$ Crop Physiology Division, Bangladesh Institute of Nuclear Agriculture, Mymensingh-2202, Bangladesh. 
farmers. At maturity, morphological parameters including yield attributes such as plant height, number of branches, siliquae and seeds per plant were recorded from randomly selected ten competitive plants. Ten central rows of each plot were harvested avoiding border effects to estimate seed yield per unit area $(\mathrm{kg} / \mathrm{ha})$. The data were then analyzed statistically following Gomez and Gomez (1984) and the mean values were compared by DMRT at 5\% level of significance.

Significant differences were observed in most of the cases amongst the mutants and control variety for the traits under evaluation at both of individual location and combined over locations under both management practices (Table 1). Two mutants RM-025 and RM2-16-96 produced the tallest plant $(98 \mathrm{~cm})$ at Ishurdi and showed non-significant difference with the control variety while the mutant RM-005 produced the shortest plant height of $84 \mathrm{~cm}$. Mutants RM-005 also produced significantly shorter plant height than the other two mutants and control variety at Jamalpur and combined over locations. Under on-farm trial at farmers' field, mutants RM-025 and RM2-16-96 and control variety produced significantly taller plant (96, 93 and $93 \mathrm{~cm}$, respectively) than the mutant RM-005. Chauhan and Kumar (1986), Shah et al. (1990) and Javed et al. (2003) isolated short statured mutants from mutagen treated populations of rapeseed and mustard. These results confirmed the present findings of obtaining short statured rapeseed mutant and also confirm that induced mutation through gamma rays had played a significant role in the alteration of plant architecture in rapeseed and mustard. Results showed that on an average, mutant RM-005 and RM- 025 produced the highest number of 2.3 primary branches/plant with statistical similarity with RM2-16-96 while control variety Binasarisha-4 produced the lowest number of 2.1 branches/plant under on-station trial. Similar trend was also observed in on-farm trial. Number of siliquae/plant, the most important component of seed yield in oilseed Brassica, showed significant differences in combined over locations and also in individual location except at Magura for onstation trial. Under on-farm trial, means combined over locations and individual location showed significant difference for this character except at Pabna. On an average, mutants RM-005 and RM025 produced the highest number of siliquae/plant ( 80 and 82, respectively) and showed significant difference from other mutant RM2-16-96 and the control variety under on-station trial. Under on-farm trial, RM-025 produced the highest number of siliquae/plant (80) having statistically different from other two mutants and the control variety Binasarisha-4. Two mutants RM-025 and RM-005 produced not only higher number of primary branches, but also higher number of siliquae/plant. Genotypes with more branches and siliqua/plant have also been reported in oilseed Brassica (Chauhan and Kumar 1986, Naz and Islam 1979, Shah et al. 1990, Javed et al. 2003, Malek and Monshi 2009) as a consequence of mutagenesis.

Among the five locations under on-station trial, only three showed statistically different number of seeds/siliqua, but combined means were statistically similar to each other. However, it was statistically similar at all the four individual locations and combined over locations under onfarm trial. Means combined over locations and also in individual location for both of on-station and on-farm trials showed significant differences for 1000-seed weight. Mutant RM-005 had the highest seed weight closely followed by RM-025 which was significantly higher than the control variety, and RM2-16-96 had the lowest seed weight. Yadav et al. (1973) and Javed et al. (2003) demonstrated that number of seeds/siliqua and 1000-seed weight directly influenced the seed yield in rapeseed and mustard. The mutant RM-005 gave the highest 1000-seed weight followed by RM-025. Both the mutants exhibited higher 1000-seed weight than the mother variety, which indicates an increase in the size of seed as a result of induced mutation. Improvement in seed size i.e., obtaining bold-seeded mutants has also been achieved earlier through induced mutations in oilseed Brassica by Chauhan and Kumar (1986) and Shah et al. (1990) which confirm the present results. 
Table 1. Mean of mutant lines and check for different characters in on-station and on-farm trials.

\begin{tabular}{|c|c|c|c|c|c|c|c|c|c|}
\hline Locations & $\begin{array}{l}\text { Mutants/ } \\
\text { varieties }\end{array}$ & $\begin{array}{l}\text { Plant } \\
\text { height } \\
(\mathrm{cm})\end{array}$ & $\begin{array}{l}\text { Primary } \\
\text { branches/ } \\
\text { plant (no.) }\end{array}$ & $\begin{array}{c}\text { Siliqua/ } \\
\text { plant } \\
\text { (no.) }\end{array}$ & $\begin{array}{c}\text { Seeds/ } \\
\text { siliqua } \\
\text { (no.) }\end{array}$ & $\begin{array}{l}\text { 1000-seed } \\
\text { wt. } \\
\text { (g) }\end{array}$ & $\begin{array}{l}\text { Days to } \\
\text { maturity }\end{array}$ & $\begin{array}{c}\text { Seed } \\
\text { yield } \\
(\mathrm{kg} / \mathrm{ha})\end{array}$ & $\begin{array}{c}\text { Seed yield } \\
\text { increase over } \\
\text { control }(\%)\end{array}$ \\
\hline \multicolumn{10}{|c|}{ On-station trial } \\
\hline $\begin{array}{l}\text { Mymen- } \\
\text { singh }\end{array}$ & $\begin{array}{l}\text { RM-005 } \\
\text { RM-025 } \\
\text { RM2-16-96 } \\
\text { Binasarisha-4 }\end{array}$ & $\begin{array}{l}77 \\
80 \\
75 \\
80\end{array}$ & $\begin{array}{l}2.5 \\
2.5 \\
2.4 \\
2.4\end{array}$ & $\begin{array}{l}73 \mathrm{~b} \\
78 \mathrm{a} \\
70 \mathrm{~b} \\
72 \mathrm{~b}\end{array}$ & $\begin{array}{l}28 \mathrm{~b} \\
29 \mathrm{a} \\
28 \mathrm{~b} \\
29 \mathrm{a}\end{array}$ & $\begin{array}{l}3.56 \mathrm{a} \\
3.53 \mathrm{ab} \\
3.47 \mathrm{c} \\
3.50 \mathrm{bc}\end{array}$ & $\begin{array}{l}88 \mathrm{~b} \\
89 \mathrm{~b} \\
88 \mathrm{~b} \\
91 \mathrm{a}\end{array}$ & $\begin{array}{l}1650 \mathrm{~b} \\
1761 \mathrm{a} \\
1655 \mathrm{~b} \\
1695 \mathrm{ab}\end{array}$ & $\begin{array}{l}-2.7 \\
3.9 \\
-2.4 \\
-\end{array}$ \\
\hline Rangpur & $\begin{array}{l}\text { RM-005 } \\
\text { RM-025 } \\
\text { RM2-16-96 } \\
\text { Binasarisha-4 }\end{array}$ & $\begin{array}{l}90 \\
92 \\
96 \\
95\end{array}$ & $\begin{array}{l}2.1 \mathrm{~b} \\
2.3 \mathrm{~b} \\
2.4 \mathrm{a} \\
1.9 \mathrm{~b}\end{array}$ & $\begin{array}{l}100 \mathrm{a} \\
103 \mathrm{a} \\
97 \mathrm{ab} \\
95 \mathrm{~b}\end{array}$ & $\begin{array}{l}28 \mathrm{c} \\
29 \mathrm{bc} \\
29 \mathrm{bc} \\
30 \mathrm{a}\end{array}$ & $\begin{array}{l}3.49 \mathrm{a} \\
3.46 \mathrm{a} \\
3.40 \mathrm{~b} \\
3.43 \mathrm{ab}\end{array}$ & $\begin{array}{l}90 \mathrm{a} \\
88 \mathrm{~b} \\
88 \mathrm{~b} \\
90 \mathrm{a}\end{array}$ & $\begin{array}{l}2550 \mathrm{a} \\
2575 \mathrm{a} \\
2300 \mathrm{~b} \\
2300 \mathrm{~b}\end{array}$ & $\begin{array}{l}10.9 \\
12.0 \\
0.0 \\
-\end{array}$ \\
\hline Magura & $\begin{array}{l}\text { RM-005 } \\
\text { RM-025 } \\
\text { RM2-16-96 } \\
\text { Binasarisha-4 }\end{array}$ & $\begin{array}{l}88 \\
90 \\
92 \\
88\end{array}$ & $\begin{array}{l}2.4 \\
2.3 \\
2.2 \\
2.2\end{array}$ & $\begin{array}{l}69 \\
67 \\
65 \\
64\end{array}$ & $\begin{array}{l}29 \mathrm{a} \\
29 \mathrm{a} \\
29 \mathrm{a} \\
28 \mathrm{~b}\end{array}$ & $\begin{array}{l}3.48 \mathrm{a} \\
3.41 \mathrm{~b} \\
3.33 \mathrm{c} \\
3.40 \mathrm{~b}\end{array}$ & $\begin{array}{l}80 \mathrm{a} \\
80 \mathrm{a} \\
78 \mathrm{~b} \\
81 \mathrm{a}\end{array}$ & $\begin{array}{l}1506 \mathrm{a} \\
1506 \mathrm{a} \\
1269 \mathrm{~b} \\
1239 \mathrm{~b}\end{array}$ & $\begin{array}{l}21.5 \\
21.5 \\
2.4 \\
-\end{array}$ \\
\hline Jamalpur & $\begin{array}{l}\text { RM-005 } \\
\text { RM-025 } \\
\text { RM2-16-96 } \\
\text { Binasarisha-4 }\end{array}$ & $\begin{array}{l}69 \mathrm{~b} \\
76 \mathrm{a} \\
79 \mathrm{a} \\
75 \mathrm{a}\end{array}$ & $\begin{array}{l}2.3 \mathrm{a} \\
2.2 \mathrm{~b} \\
2.1 \mathrm{~b} \\
2.0 \mathrm{~b}\end{array}$ & $\begin{array}{l}66 \mathrm{~b} \\
72 \mathrm{a} \\
64 \mathrm{bc} \\
60 \mathrm{c}\end{array}$ & $\begin{array}{l}27 \mathrm{~b} \\
28 \mathrm{ab} \\
29 \mathrm{a} \\
27 \mathrm{~b}\end{array}$ & $\begin{array}{l}3.51 \mathrm{a} \\
3.45 \mathrm{a} \\
3.35 \mathrm{c} \\
3.42 \mathrm{~b}\end{array}$ & $\begin{array}{l}88 \mathrm{a} \\
86 \mathrm{~b} \\
88 \mathrm{a} \\
87 \mathrm{ab}\end{array}$ & $\begin{array}{l}1518 \mathrm{~b} \\
1653 \mathrm{a} \\
1540 \mathrm{~b} \\
1495 \mathrm{~b}\end{array}$ & $\begin{array}{l}1.5 \\
10.6 \\
3.0 \\
-\end{array}$ \\
\hline Ishurdi & $\begin{array}{l}\text { RM-005 } \\
\text { RM-025 } \\
\text { RM2-16-96 } \\
\text { Binasarisha-4 }\end{array}$ & $\begin{array}{l}84 \mathrm{~b} \\
98 \mathrm{a} \\
98 \mathrm{a} \\
96 \mathrm{a}\end{array}$ & $\begin{array}{l}2.3 \mathrm{a} \\
2.3 \mathrm{a} \\
2.2 \mathrm{ab} \\
2.1 \mathrm{~b}\end{array}$ & $\begin{array}{l}93 \mathrm{a} \\
88 \mathrm{ab} \\
85 \mathrm{bc} \\
83 \mathrm{c}\end{array}$ & $\begin{array}{l}30 \\
31 \\
31 \\
30\end{array}$ & $\begin{array}{l}3.52 \mathrm{a} \\
3.49 \mathrm{a} \\
3.31 \mathrm{~b} \\
3.31 \mathrm{~b}\end{array}$ & $\begin{array}{l}83 \\
83 \\
84 \\
84\end{array}$ & $\begin{array}{l}2234 \mathrm{a} \\
2230 \mathrm{a} \\
2013 \mathrm{~b} \\
1988 \mathrm{~b}\end{array}$ & $\begin{array}{l}12.4 \\
12.2 \\
1.3 \\
-\end{array}$ \\
\hline \multicolumn{10}{|c|}{ Combined means over five locations } \\
\hline & $\begin{array}{l}\text { RM-005 } \\
\text { RM-025 } \\
\text { RM2-16-96 } \\
\text { Binasarisha-4 }\end{array}$ & $\begin{array}{l}81 \mathrm{~b} \\
87 \mathrm{a} \\
88 \mathrm{a} \\
87 \mathrm{a}\end{array}$ & $\begin{array}{l}2.3 \mathrm{a} \\
2.2 \mathrm{~b} \\
2.3 \mathrm{a} \\
2.1 \mathrm{c}\end{array}$ & $\begin{array}{l}80 \mathrm{a} \\
82 \mathrm{a} \\
76 \mathrm{~b} \\
75 \mathrm{~b}\end{array}$ & $\begin{array}{l}29 \\
29 \\
29 \\
29\end{array}$ & $\begin{array}{l}3.51 \mathrm{a} \\
3.47 \mathrm{a} \\
3.37 \mathrm{~b} \\
3.41 \mathrm{~b}\end{array}$ & $\begin{array}{l}86 \mathrm{ab} \\
85 \mathrm{~b} \\
85 \mathrm{~b} \\
87 \mathrm{a}\end{array}$ & $\begin{array}{l}1892 \mathrm{a} \\
1945 \mathrm{a} \\
1755 \mathrm{~b} \\
1743 \mathrm{~b}\end{array}$ & $\begin{array}{l}8.5 \\
11.6 \\
0.0\end{array}$ \\
\hline \multicolumn{10}{|c|}{ On-farm trial } \\
\hline $\begin{array}{l}\text { Mymen- } \\
\text { singh }\end{array}$ & $\begin{array}{l}\text { RM-005 } \\
\text { RM-025 } \\
\text { RM2-16-96 } \\
\text { Binasarisha-4 }\end{array}$ & $\begin{array}{l}83 \mathrm{~b} \\
99 \mathrm{a} \\
98 \mathrm{a} \\
96 \mathrm{a}\end{array}$ & $\begin{array}{l}2.5 \\
2.7 \\
2.4 \\
2.5\end{array}$ & $\begin{array}{l}55 \mathrm{~b} \\
67 \mathrm{a} \\
61 \mathrm{ab} \\
56 \mathrm{~b}\end{array}$ & $\begin{array}{l}28 \\
26 \\
27 \\
27\end{array}$ & $\begin{array}{l}3.46 \mathrm{a} \\
3.42 \mathrm{a} \\
3.29 \mathrm{~b} \\
3.31 \mathrm{~b}\end{array}$ & $\begin{array}{l}91 \mathrm{a} \\
88 \mathrm{~b} \\
91 \mathrm{a} \\
92 \mathrm{a}\end{array}$ & $\begin{array}{l}1205 \mathrm{~b} \\
1325 \mathrm{a} \\
1213 \mathrm{~b} \\
1175 \mathrm{~b}\end{array}$ & $\begin{array}{l}2.6 \\
12.8 \\
3.2 \\
-\end{array}$ \\
\hline Rangpur & $\begin{array}{l}\text { RM-005 } \\
\text { RM-025 } \\
\text { RM2-16-96 } \\
\text { Binasarisha-4 }\end{array}$ & $\begin{array}{l}70 \mathrm{~b} \\
82 \mathrm{a} \\
83 \mathrm{a} \\
80 \mathrm{a}\end{array}$ & $\begin{array}{l}2.6 \\
2.6 \\
2.3 \\
2.3\end{array}$ & $\begin{array}{l}92 \mathrm{~b} \\
97 \mathrm{a} \\
86 \mathrm{c} \\
91 \mathrm{~b}\end{array}$ & $\begin{array}{l}31 \\
30 \\
31 \\
31\end{array}$ & $\begin{array}{l}3.51 \mathrm{a} \\
3.46 \mathrm{a} \\
3.34 \mathrm{c} \\
3.40 \mathrm{~b}\end{array}$ & $\begin{array}{l}90 \mathrm{ab} \\
90 \mathrm{ab} \\
88 \mathrm{~b} \\
92 \mathrm{a}\end{array}$ & $\begin{array}{l}2424 \mathrm{ab} \\
2534 \mathrm{a} \\
2100 \mathrm{~b} \\
2249 \mathrm{~b}\end{array}$ & $\begin{array}{l}7.8 \\
12.7 \\
-6.6 \\
-\end{array}$ \\
\hline Magura & $\begin{array}{l}\text { RM-005 } \\
\text { RM-025 } \\
\text { RM2-16-96 } \\
\text { Binasarisha-4 }\end{array}$ & $\begin{array}{l}77 \mathrm{~b} \\
88 \mathrm{a} \\
87 \mathrm{a} \\
90 \mathrm{a}\end{array}$ & $\begin{array}{l}2.9 \\
3.2 \\
3.0 \\
2.9\end{array}$ & $\begin{array}{l}56 \mathrm{~b} \\
65 \mathrm{a} \\
57 \mathrm{~b} \\
55 \mathrm{~b}\end{array}$ & $\begin{array}{l}26 \\
27 \\
27 \\
27\end{array}$ & $\begin{array}{l}3.50 \mathrm{a} \\
3.47 \mathrm{a} \\
3.40 \mathrm{~b} \\
3.43 \mathrm{~b}\end{array}$ & $\begin{array}{l}82 \mathrm{ab} \\
83 \mathrm{a} \\
81 \mathrm{~b} \\
83 \mathrm{a}\end{array}$ & $\begin{array}{l}1209 \mathrm{~b} \\
1464 \mathrm{a} \\
1229 \mathrm{~b} \\
1206 \mathrm{~b}\end{array}$ & $\begin{array}{l}0.0 \\
21.4 \\
1.9 \\
-\end{array}$ \\
\hline Pabna & $\begin{array}{l}\text { RM-005 } \\
\text { RM-025 } \\
\text { RM2-16-96 } \\
\text { Binasarisha-4 }\end{array}$ & $\begin{array}{l}87 \mathrm{~b} \\
113 \mathrm{a} \\
113 \mathrm{a} \\
107 \mathrm{a}\end{array}$ & $\begin{array}{l}3.6 \mathrm{a} \\
3.5 \mathrm{a} \\
3.3 \mathrm{a} \\
2.6 \mathrm{~b}\end{array}$ & $\begin{array}{l}92 \\
90 \\
87 \\
87\end{array}$ & $\begin{array}{l}31 \\
30 \\
31 \\
30\end{array}$ & $\begin{array}{l}3.58 \mathrm{a} \\
3.53 \mathrm{~b} \\
3.49 \mathrm{~b} \\
3.50 \mathrm{~b}\end{array}$ & $\begin{array}{l}86 \mathrm{~b} \\
85 \mathrm{~b} \\
88 \mathrm{ab} \\
90 \mathrm{a}\end{array}$ & $\begin{array}{l}2061 \mathrm{a} \\
1971 \mathrm{ab} \\
1888 \mathrm{~b} \\
1925 \mathrm{~b}\end{array}$ & $\begin{array}{l}7.1 \\
2.4 \\
-1.9 \\
-\end{array}$ \\
\hline \multicolumn{10}{|c|}{ Combined means over four locations } \\
\hline & $\begin{array}{l}\text { RM-005 } \\
\text { RM-025 } \\
\text { RM2-16-96 } \\
\text { Binasarisha-4 }\end{array}$ & $\begin{array}{l}79 \mathrm{~b} \\
96 \mathrm{a} \\
93 \mathrm{a} \\
93 \mathrm{a}\end{array}$ & $\begin{array}{l}2.9 \mathrm{ab} \\
3.0 \mathrm{a} \\
2.8 \mathrm{~b} \\
2.6 \mathrm{c}\end{array}$ & $\begin{array}{l}74 \mathrm{~b} \\
80 \mathrm{a} \\
73 \mathrm{~b} \\
72 \mathrm{~b}\end{array}$ & $\begin{array}{l}29 \\
28 \\
29 \\
29\end{array}$ & $\begin{array}{l}3.51 \mathrm{a} \\
3.47 \mathrm{a} \\
3.38 \mathrm{~b} \\
3.41 \mathrm{~b}\end{array}$ & $\begin{array}{l}87 \mathrm{~b} \\
87 \mathrm{~b} \\
87 \mathrm{~b} \\
89 \mathrm{a}\end{array}$ & $\begin{array}{l}1725 \mathrm{ab} \\
1824 \mathrm{a} \\
1608 \mathrm{~b} \\
1639 \mathrm{~b}\end{array}$ & $\begin{array}{l}5.2 \\
11.3 \\
-1.9 \\
-\end{array}$ \\
\hline
\end{tabular}

Same letter(s) in a column did not differ significantly at $\mathrm{p} \leq 0.05$ by DMRT. 
Among the mutants, RM-025 showed the highest seed yield performance in individual location and combined over locations in on-station trial. On an average, RM-025 gave seed yield of $1945 \mathrm{~kg} / \mathrm{ha}$ followed by RM-005 (1892 kg/ha) which was 11.6 and $8.5 \%$ higher than the control variety, Binasarisha-4 (1743 kg/ha). Similar trend was also observed under on-farm trial both at individual location and combined over locations. On an average, RM-025 produced the highest seed yield of $1824 \mathrm{~kg} / \mathrm{ha}$ followed by RM-005 (1725 kg/ha) which was 11.3 and 5.2\% higher than the mother variety, respectively.

Induction of early maturity is one of the most frequent characters modified in the mutation breeding experiments in many crops including oilseed Brassica. Results showed that Binasarisha4 required the longest period to mature ( 87 days) while all the three mutants required significantly lower period ( 85 days) in on-station trial. Under on-farm trial, Binasarisha-4 also required significantly higher maturity period (89 days) than the mutants (87 days). Development of several early maturity mutants than the mother has also been reported in rice (Miah et al. 1981), in soybeans (Zakri 1991) and in rapeseed (Das et al. 1999, Malek and Monshi 2009) which confirm the present result.

A little variation was observed in the yield and yield components between two managements and within different locations also. This was due to the prevailing environmental factors.

It was concluded that the overall performance of the mutants and control variety for the yield and yield components indicates that two mutants RM-025 and RM-005, because of its higher seed yield potential along with higher number of primary branches and siliquae/plant, and 1000-seed weight, hold promise to be mutant varieties. Moreover, this suggests that gamma rays irradiation can be fruitfully applied to develop new varieties with high yield and other improved agronomic traits in oleiferous Brassica.

\section{References}

BBS 2010. Monthly Statistical Bulletin-Bangladesh. Bangladesh Bureau of Statistics. Statistics Division, Ministry of Planning, Government of the People's Republic of Bangladesh. June 68 p.

Chauhan YS and K Kumar 1986. Gamma ray induced chocolate B seeded mutant in Brassica campestris L. cv. Yellow Sarson. Current Sci. India 55: 410.

Das ML, A Rahman and MA Malek 1999. Two early maturing and high yielding varieties of rapeseed developed through induced mutation technique. Bangladesh J. Bot. 28(1): 27-33.

Gomez KA and AA Gomez 1984. Statistical Procedures for Agricultural Research. John Wiley and Sons, USA.

Javed MA, MA Siddiqui, MKR Khan, A Khatri, IA Khan, NA Dehar, MH Khanzada and R Khan 2003. Development of high yielding mutants of Brassica campestris L. cv. Toria selection through gamma rays irradiation. Asian J. Plant Sci. 2(2): 192-195.

Malek MA and FI Monshi 2009. Performance evaluation of rapeseed mutants. Bangladesh J. Agril. Sci. 36(1): 81-84.

Miah AJ, MA Mansur and MJ Uddin 1981. Improvement of rice through induced mutations. Indian J. Agric. Sci. 51(3): 145-146.

Naz RMA and RZ Islam 1979. Effect of irradiation on Brassica trilocularis (yellow sarson). Pak. J. Bot. 17: 87-93.

Shah SA, I Ali and S Rahman 1990. Induction and selection of superior genetic variables of oilseed rape, Brassica napus L. The Nucleus 7: 37-40.

Yadav TP, H Singh, VP Gupta and RK Rana 1973. Heterosis and combining ability in raya for yield and its components. Indian J. Genet. Pl. Breed. 34: 684-695.

Zakri AH 1991. Breeding high yielding soybean varieties using induced mutation. In: Mutation Breeding for Crop Improvement. Proc. Symp., FAO/IAEA, Vienna, 18-22 June, 1990. 2: 163-170. 